\title{
研究全身麻醉和蛛网膜下腔麻醉对老年骨科手术 患者的麻醉效果
}

\section{To investigate the effect of general anesthesia and subarachnoid anesthesia on elderly patients undergoing orthopaedic surgery}

\author{
毛芹 \\ Qin Mao \\ 徐州市仁慈医院 中国・江苏徐州 221700 \\ Xuzhou Mercy Hospital, Xuzhou,Jiangsu, 221700, China
}

\begin{abstract}
摘 要: 目的:对比研究全身麻醉和蛛网膜下腔麻醉对老年骨科手术患者的麻醉效果。方法: 将我院在 2018 年 3 月至 2020 年 6 月行骨科手术的老年患者 48 例作为研究对象,按照双色球分组的方式,将其划分为对照组 $(n=24$, 在术中给予全身麻醉) 和观察组 $(n=24$, 在术中给予蛛网膜下腔麻醉)。对两组患者术后恢复指标进行观察。结果: 观察组患者的术后苏醒时间、语 言恢复时间相较于对照组, 差异显著 $(\mathrm{P}<0.05)$ 。结论: 在老年骨科手术实施时, 对患者给予蛛网膜下腔麻醉和全身麻醉会存 在不同的差异, 且以蛛网膜下腔麻醉占优, 值得临床选用和推广。
\end{abstract}

\begin{abstract}
Objective: To compare the anesthetic effect of general anesthesia and subarachnoid anesthesia on elderly orthopaedic surgery patients. Methods: a total of 48 elderly patients who underwent orthopedic surgery in our hospital from March 2018 to June 2020 were selected as study subjects. According to the the double chromosphere grouping method, they were divided into the control group ( $n=24$, general anesthesia was given during the operation) and the observation group $(n=24$, subarachnoid anesthesia was given during the operation). The postoperative recovery indexes of the two groups were observed. Results: Compared with the control group, the postoperative waking time and speech recovery time of the observation group were significantly different $(\mathrm{P}<0.05)$. Conclusion: There are differences between subarachnoid anesthesia and general anesthesia in elderly patients undergoing orthopedic surgery, and subarachnoid anesthesia is superior, which is worthy of clinical selection and promotion.
\end{abstract}

关键词: 全身麻醉;蛛网膜下腔麻醉; 老年患者;骨科手术;麻醉效果

Keywords : General anesthesia; Subarachnoid anesthesia; Elderly patients ; Orthopaedic surgery; Anesthesia effect

DOI : $10.36012 /$ pmr. v2i6. 2967

随着个体年龄的增长, 机体会出现退行性变化, 从而使 得老人的免疫力降低,疾病发生可能性较高。骨质疏松症作 为临床常见的一种老年疾病, 其使得老人群体在日常生活与 生产中, 一旦受到外力干扰, 发生骨折的可能性提升, 这也是 当前老年骨折患者数量增多的主要原因。当然, 老年群体还 会出现其他骨病变, 这些都需要进入骨科治疗。在当前, 骨 科收治的老年患者数量增多, 且多数都需要行手术治疗。但
由于老年群体生理特点的特殊性, 在相关研究中指出, 对老 年骨科患者行麻醉干预后, 可能会使得其脑部血流量减少, 造成神经系统与中枢神经元受损的可能性较高, 且患者术后 清醒时间与功能恢复时间较长, 这些都会对手术造成极大的 限制 ${ }^{[1]}$ 。全身麻醉与蛛网膜下腔麻醉是骨科手术的, 常见两 种麻醉形式。本次研究结合其对老年骨科手术患者的恢复 影响进行探讨, 详细报道如下。

【作者简介】毛芹 (1978 ), 女, 民族, 汉族, 江苏徐州丰县人, 本科, 主治医师, 从事临床麻醉研究。 


\section{1 一般资料与方法}

\section{1 一般资料}

将我院在 2018 年 3 月至 2020 年 6 月行骨科手术的老年 患者 48 例作为研究对象, 按照双色球分组的方式, 将其划分 为对照组和观察组, 每组 24 例。

纳入标准: (1) 所有患者年龄>60 岁, 且符合临床骨科手 术的实施指征 ${ }^{[2]} ;$; (2) 患者无严重的器质性缺损与认知障 碍; (3) 研究经过患者与其家家属的同意。

对照组中, 男性患者 12 例, 女性患者 12 例, 年龄在 $60 \sim$ 85 岁的区间内, 平均年龄 $(70.34 \pm 3.24)$ 岁;

观察组中, 男性患者 14 例, 女性患者 10 例, 年龄在 $60 \sim$ 86 岁的区间内,平均年龄 $(70.12 \pm 3.56)$ 岁。

对两组患者的一般资料进行整理, $\mathrm{P}>0.05$, 可比。

\section{2 方法}

在患者经过常规检查, 拟定手术时间后, 术前, 未给予任 何药物。对照组患者采取全身麻醉, 其以静脉注射咪达唑仑 $0.04 \mathrm{mg} / \mathrm{kg}+$ 芬太尼 $5 \mathrm{ug} / \mathrm{kg}+$ 依托咪酯 $0.3 \mathrm{mg} / \mathrm{kg}+$ 顺阿曲库胺 $0.15 \mathrm{mg} / \mathrm{kg}$ 来作为麻醉诱导, 然后, 行气管插管。使用麻醉 呼吸机, 来对患者进行机械通气, 并且运用靶控输注的方式, 来给予异丙酚+瑞芬太尼进行维持麻醉。在手术过程中, 需 要结合患者的实际状况, 来适当的进行顺阿曲库胺追加, 以 保持患者肌肉松弛的状态。

观察组患者行蛛网膜下腔麻醉干预, 腰椎间隙来进行穿 刺, 并置入置管, 采取蛛网膜下腔进行麻醉。使得麻醉平面 尽可能处于 $\mathrm{T} 10$ 以下。在手术实施的过程中, 可根据患者的 主诉适当的追加罗哌卡因, 且根据患者的体征, 来对其进行 麻醉深度调整。

\section{3 评价指标}

对两组患者术后语言恢复时间与苏醒时间进行统计。

\section{4 统计学分析}

数据以统计学软件 SPSS18.0 分析, 以 $(\overline{\mathrm{x}} \pm \mathrm{s})$ 表示计量资 料, 经 $\mathrm{t}$ 检验; 以率 $(\%)$ 表示计数资料, 经 $\chi^{2}$ 检验, $\mathrm{P}<0.05$ 为 差异有统计学意义。

\section{2 结果}

观察组患者的术后苏醒时间、语言恢复时间相较于对照 组, 差异显著 $(\mathrm{P}<0.05)$ 。详见下表。

表 12 组患者的语言恢复时间与苏醒时间比较 $(\bar{x} \pm \mathrm{s}, \mathrm{min})$

\begin{tabular}{c|c|c|c}
\hline 组别 & 例数 & 苏醒时间 & 语言恢复时间 \\
\hline 观察组 & 24 & $3.76 \pm 2.38$ & $6.21 \pm 3.42$ \\
\hline 对照组 & 24 & $11.82 \pm 3.58$ & $14.58 \pm 5.02$ \\
\hline
\end{tabular}

\section{3 讨论}

在当前, 我国基本进入了人口老龄化阶段,这也就意味 着老年人口基数较大。而老年群体的身体和心理状态存在 一定的特殊性, 其疾病的发生率较高 ${ }^{[3]}$ 。在目前, 以骨科收 治的老年患者行手术治疗的频率较高, 但在相关研究中指 出, 骨科手术实施过程中, 麻醉的使用, 可能会对患者的脑部 供血造成限制, 从而使得大脑代谢处于异常状态, 很容易导 致术后恢复状况较差。再加上老年患者本身处于各项功能 退化阶段,在受到麻醉药物的影响后, 极有可能会出现神经 功能障碍。尤其是全身麻醉, 其在实施的过程中, 需要维持 追加麻醉药物, 对患者的全身都存在影响, 这就使得其麻醉 的影响程度加深 ${ }^{[4]}$ 。在这种状况下, 骨科在对老年患者实施 手术治疗时, 开始注重蛛网膜下腔麻醉的应用。蛛网膜下空 麻醉从本质上来说是一种局部麻醉形式, 能够针对患者的病 灶与手术区间, 来选择合适的神经区域作为麻醉对象, 即在 实施后, 基本上不会对患者的大脑产生限制 ${ }^{[5]}$ 。从本次研究 结果上来看, 骨科老年患者实施蛛网膜下腔麻醉后的苏醒时 间与语言功能恢复时间相对于全身麻醉更短, 这也就意味 着,其对于老年患者的影响较弱。

综上所述,在老年骨科手术实施时, 对患者给予蛛网膜 下腔麻醉和全身麻醉会存在不同的差异, 且以蛛网膜下腔麻 醉占优,值得临床选用和推广。

\section{参考文献}

１１］王丽丽,杨慧. 全身麻醉和蛛网膜下腔麻醉对老年骨科手术患 者术后短期认知功能的影响研究 $[\mathrm{J}]$. 中国保健营养, 2020,30 (20) : 160 .

２］窦以清. 全身麻醉和蛛网膜下腔麻醉对老年骨科手术患者术 后短期认知功能的影响 $[\mathrm{J}]$. 健康之友, 2020, (5) : 155 .

３３］李增. 分析采用不同麻醉方式对老年骨科手术后患者短期认 知功能与睡眠状况的影响 $[\mathrm{J}]$. 世界最新医学信息文摘 ( 连续 型电子期刊) $, 2020,20(4): 82,87$.

[4] 姚有干,王寿根. 全身麻醉和蛛网膜下腔麻醉对老年骨科手术 患者术后短期认知功能的影响 $[\mathrm{J}]$. 医学美学美容, 2019,28 (22) :30-31

［5］付珍红. 全身麻醉与蛛网膜下腔麻醉在老年骨科患者手术治 疗中的 应用及术后短期认知功能变化分析 $[\mathrm{J}]$. 山西医药杂 志 $, 2018,47(2): 171-174$. 